\title{
Sum frequency vibrationnal spectroscopy of water molecules at interfaces
}

\author{
E. Freysz, Q. Du* and Y.R. Shen*
}

Centre de Physique Moléculaire Optique et Hertzienne, Université Bordeaux 1, 351 Cours de la Libération, 33405 Talence cedex, France

* Department of Physics, University of California, Materials Sciences Division, Lawrence Berkeley Laboratory, Berkeley, California 94720, U.S.A.

\begin{abstract}
We present the surface vibrationnal sum frequency spectroscopy technique and we illustrate the ability of this method to analyze the properties of water at different interfaces. We stress the originality of this spectroscopy to evidence water structure at hydrophobic interfaces and discuss the extension of the sum frequency generation spectroscopy.
\end{abstract}

\section{INTRODUCTION}

Buried water interfaces play an important role in many surface science phenomena. For instance, structure and properties of water at various interfaces is fundamental for understanding of various phenomena. It range from electrochemistry to biological processes such as membranes stability and proteins activity. However understanding of water interfacial properties is still rather primitive even though it is crucial for advances of many discipline of science as well as for the control of many technological processes. In this paper we will summarize our results on structure and properties of water at various interfaces $[1,2,3]$. We will show that this work was made possible by the original use of surface vibrationnal sum frequency generation (SFG). In the first part of the paper we will rapidly present the basic ideas of this technic. In the second part, we will present the experimental set-up we used and we will discuss the main results we obtained.

\section{BASIC THEORY}

Infrared-visible sum frequency generation (SFG) as a technic for surface vibrationnal spectroscopy has been described in earlier publication [4]. It is dipole forbidden in a centrosymmetric medium but allowed at an interface where the inversion symmetry is broken. The material parameter that can effectively be deduced from the measurement of SFG is the second order susceptibility $\chi_{s}^{(2)}\left(\omega_{s f}=\omega_{v}+\omega_{i r}\right)$ which is mainly due to electric dipole contribution from an oriented surface layer. Indeed when two intense light fields are simultaneously incident on an interface, they induced a nonlinear polarization at the interface and in the bulk material which may subsequently radiate. The surface dipole polarization is written as:

$$
P\left(\omega_{s}\right)=\chi^{(2)}\left(\omega_{s}=\omega_{1}+\omega_{2}\right): E\left(\omega_{1}\right) E\left(\omega_{2}\right)
$$


where $E_{1}$ and $E_{2}$ are the local fields in the monolayer. The microscopic expression of $\chi^{(2)}$ can be obtained from second order perturbation theory [ 5 ]. In the case of monolayer with appreciable second order susceptibility deposited on a centrosymmetric isolator, the monolayer dipole susceptibility often dominates other contribution since the $\chi^{(2)}=0$ for centrosymmetric isolator. The surface susceptibility can then be related to the nonlinear molecular polarizability $\alpha_{k l m}^{(2)}$ by a coordinate transformation averaged over the molecular orientationnal distribution

$$
\chi_{i j k}^{(2)}=N_{s}((i . l)(j . m)(k . n)) \alpha_{l m n}^{(2)}
$$

where Ns is the surface molecular density and the angular brackets denotes the orientationnal average. The independent elements of $\chi^{(2)}$ can be deduced by measuring the signal at the sum frequency for different polarizations of the incident and output beams. If $\alpha^{(2)}$ is known, the parameters of an orientationnal distribution can be deduced. In the case of resonant infrared non-resonant visible SFG, the nonlinear molecular polarizability near resonance can be written as a sum of a resonant and non resonant term:

$$
\begin{gathered}
\alpha^{(2)}=\alpha_{N R}^{(2)}+\alpha_{R}^{(2)} \\
a_{R}^{(2)}=\sum_{q} \frac{A_{q}}{\left(\omega_{q}-\omega_{i r}-i \Gamma_{q}\right)}
\end{gathered}
$$

where $A_{q}, \omega_{q}$, and $\Gamma_{q}$ are the strength, resonant frequency, and damping constant of the $q_{\text {th }}$ vibrationnal mode of the molecules. The resonant enhancement of $\alpha(2)$ and therefore of $\chi^{(2)}$ as $\omega_{\mathrm{ir}}$ scans over $\omega_{\mathrm{q}}$ then yields the surface vibrationnal spectrum observed by SFG.

\section{APPLICATIONS}

\subsection{Experimental set-up}

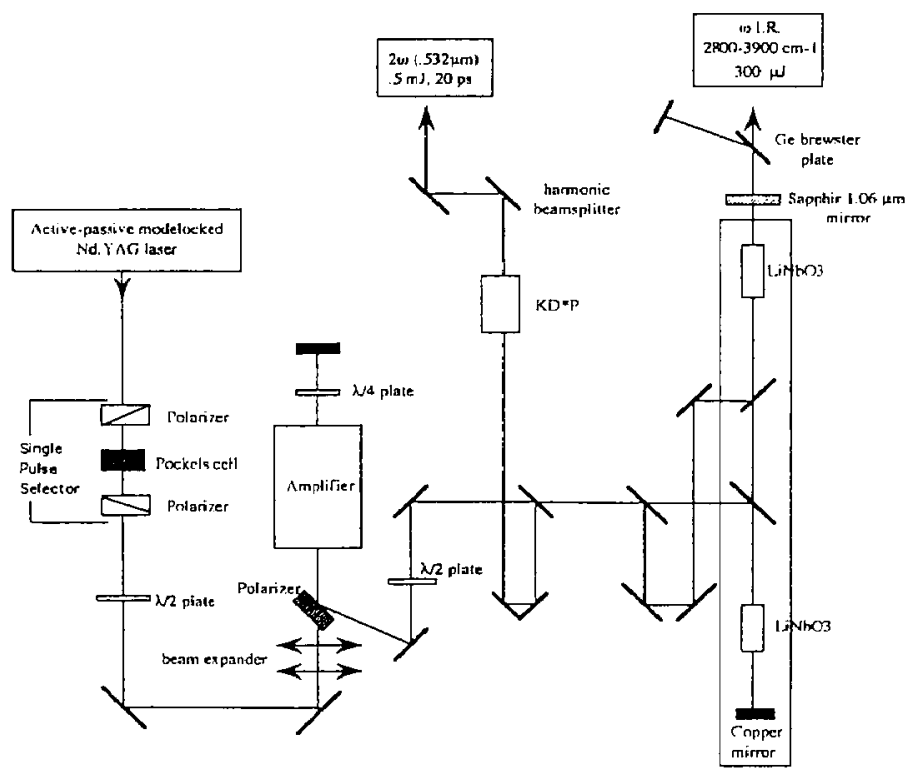

Figure 1: Experimental set-up 
A schematic of the experimental set-up is presented on figure 1. It includes an active passive mode locked laser producing $35 \mathrm{ps}$ pulses at a repetition rate of $10 \mathrm{~Hz}$. A single pulse is selected from the Q-switched pulse train with a fast pockels cell in combination with crossed polarizers. The single pulse is amplified in a double passes amplifier to obtain $20 \mathrm{~mJ}$. About $4 \mathrm{~mJ}$ is split off to be doubled in a $\mathrm{KD}^{*} \mathrm{P}$ crystal to produce about $0.5 \mathrm{~mJ}$ of $532 \mathrm{~nm}$ light. The rest is send to a dual crystal LiNbO3 optical parametric generator and amplifier to produce infrared pulses of approximately $300 \mu \mathrm{J}$ tunable from 2700 to $3900 \mathrm{~cm}^{-1}$. After this set-up, the visible beam goes through a lens, a polarizer and then a half wave plate to control polarization at sample. The Infrared beam goes through Fresnel rhomb which serves as a broad band half wave plate and then passes through a focusing lens. Beams are overlapped on the sample to a spot size about $300 \mu \mathrm{m}$ in diameter. For the SFG measurements, we used a $0.5 \mathrm{~mJ}$ of visible laser pulses at 532 $\mathrm{nm}$ and $0.2 \mathrm{~mJ}$ infrared pulses tunable around the broad $\mathrm{OH}$ stretching frequency of water from $2700-3900 \mathrm{~cm}^{-1}$. The infrared and visible beams were incident at an angle around $50^{\circ}$ and overlap at the interface. For water surface spectroscopy, we used a Teflon cell filled with ultra pure water with a resistivity higher than $18 \mathrm{M} \Omega / \mathrm{cm}$, obtained from a Millipore filtration system. For quartz-water interface spectrum, we used infrared fused quartz windows. All SFG spectra were normalized to SFG signal reflected from quartz crystal in order to reduce the effects of laser fluctuation; change of beam overlap, and IR. absorption from the fused quartz windows.

\subsection{Air-water interface}

The study of pure liquid-vapor interfaces has had a long history [6]. A large amount of theoretical work on the subject already exists in the literature [7]. Experiment, however have been limited to measurement of thermodynamic quantities such as surface tension and surface electrical potential [8]. They can provide only a crude information on the molecular orientation at an interface. Recent, second harmonic generation (SHG) study the air-water interface conclude that water molecules at this interface were polar oriented, but interpretation of the results were critically dependent on the temperature dependence of the observed signal [9]. Since, as a rule, SFG is more sensitive than SHG we carried out the study of the air-water interface. In particular, SFG spectroscopy yields vibrationnal spectra of surface molecules that allows to identify species and obtain detailed information on the structure and orientation of particular atomic group within the molecules.

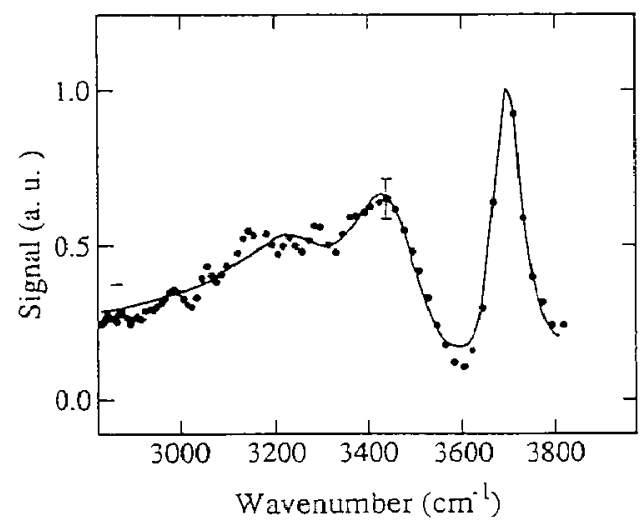

Figure 2: SFG spectrum of the air-water interface: $(\bullet)$ Data (-) Fit 
Figure 2 shows the SFG spectrum of water at the air-water interface for ssp polarization combination (SF output, visible input, and IR. input are respectively $s, s$ and p polarized). The signal with other polarization was less than $10 \%$ of the ssp peak signal and exhibited hardly any discernible resonant features within our experimental uncertainty. This spectrum can be reasonably well fitted assuming three vibrationnal resonances around 3200,3400 and $3700 \mathrm{~cm}^{-1}$. The spectrum shows two main features: a broad band between 3100 and $3500 \mathrm{~cm}^{-1}$ and a relative sharp peak at $3690 \mathrm{~cm}^{-1}$. This peak can be assigned to the stretch mode of a free $\mathrm{OH}$ [10]. It structure indicates that only one $\mathrm{OH}$ of the surface water molecule pointing toward the air can be non-hydrogen bonded. If both $\mathrm{OH}$ were free two sharp peaks would appear in the spectrum: one above $3700 \mathrm{~cm}-1$, the other around $3600 \mathrm{~cm}^{-1}$ [10]. This assignment is further supported by the following observation: the free $\mathrm{OH}$ peak in the SFG spectrum disappears while the bonded $\mathrm{OH}$ bound becomes narrower and peaks towards the lower frequency side when the water surface is covered by a monolayer of long alcohol molecules which are hydrogen bounded to surface water molecules (Fig. 3a). The existence at air-water interface of water molecules with one free $\mathrm{OH}$ is actually not so surprising: water molecules prefer to establish hydrogen bonds with their nearest neighbors in a tetrahedral bounding geometry. This arrangement is not possible for surface water molecules, so a fraction of this hydrogen bonds must be broken.

We can deduced information about the free $\mathrm{OH}$ orientation and number of molecules with one free $\mathrm{OH}$ bond at the interface. Our estimation shows that the angle $\Theta$ between the free $\mathrm{OH}$ bond and the surface normal was $\leq 38^{\circ}$ [1]. This computation assumes a delta function for the orientationnal distribution. By calibrating the signal at the free $\mathrm{OH}$ resonance against the signal from a quartz signal we concluded that $20 \%$ of surface water molecules at the air-water interface have one free $\mathrm{OH}$ bond. Our estimation was in variance with the results predicted by some molecular dynamics simulations. However simple arguments leads to similar estimation: assuming maximization of total number of hydrogen bonds each water molecules possesses leads a configuration with about one dangling bond per four water molecules at the interface [3].

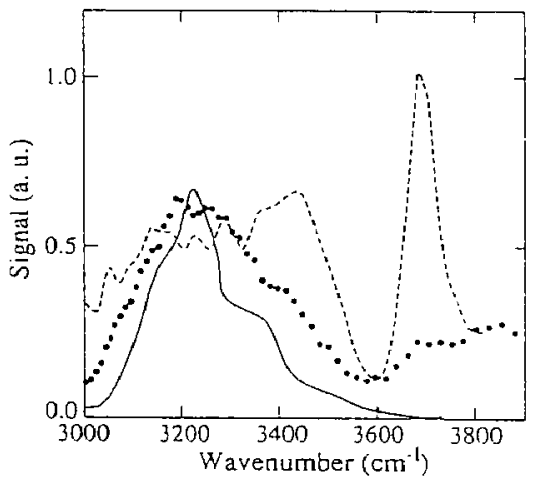

Figure 3: Spectrum of air-water interface (- - ), air-alcohol-water interface $(\bullet)$ and quartz-ice interface (__

The SFG spectrum of a water surface covered by a full monolayer in Figure 3 is also quite interesting. It has recently be shown that such a monolayer on water is able to induce an increase of the icing temperature of the nearby water because of the ordered $\mathrm{OH}$ groups in the alcohol monolayer closely match those of the hexagonal ice layer in the $a-b$ plane [11]. The similarity of our spectrum of the air-alcohol-water interface and the SFG spectrum of quartz-Ice surface, shown in Fig. 3 leads us to believe that ice nucleation by the alcohol monolayer can indeed occur. The top layer of water molecules is converted in an ice-like structure which provides a seed for ice nucleation. 
We have also studied the temperature dependence of the SFG spectrum of the airwater interface. We observed no significant change over the temperature range from 10 to $80^{\circ} \mathrm{C}$. Our results were at variance with the strong temperature dependence of the $\mathrm{OH}$ orientation predicted by some molecular dynamic simulation [12] and the SHG measurement on the vapor-water interface previously reported by Goh et al [9]. After repeating this SHG experiment this group observed a weaker change in the SHG signal which were in better agreement with our results [1].

\subsection{Study of hydrophobicity}

Wetting or non wetting is a familiar phenomena, but there is not yet a clear physical picture of the phenomena at the molecular level [13]. Moreover structure of water near hydrophobic surfaces is crucial in many different domains. Hydrophobic interaction are responsible for the formation of micelles [14] and play an important role in organizing constituent molecules of living matter into complex structure such as membranes [15]. The understanding of hydrophobic interface can also provide a better picture of how hydrophobic solute particles are surrounded by water molecules when immersed in water. The importance of such an interface has stimulated a number of theoretical studies done by numerical simulation. Experimental research on the topic, however, has been rare are non existent because of the lack of suitable techniques. We used surface vibrationnal spectroscopy by sum frequency generation to study hydrophobicity at the molecular level on two interfaces: water-hydrophobic quartz, waterhexane and we have compared them with the air-water SFG spectra (Fig. 2).

On figure 4 we report the sum frequency vibrationnal spectra made on these two hydrophobic interfaces for ssp polarization combination. The hydrophobicity of the quartz surface was obtained by depositing a monolayer of OTS ( octadecyltrichlorosilane, $\left.\mathrm{CH}_{3}\left(\mathrm{CH}_{2}\right)_{17} \mathrm{SiCl}_{3}\right)$ on the surface by mean of standard self assembly technique [16]. The hydrophobicity of this interface came from the layer of closely packed hydrocarbon chain. The hexane-water interface was obtained by spreading a thin layer of hexane on water surface. Notice the spectra is similar to the one obtain from the air-water interface presented in Fig. 2. In both case the spectrum exhibit a sharp peak around $3700 \mathrm{~cm}^{-1}$ assigned to the non-hydrogen bonded $\mathrm{OH}$. Hydrophobicity is therefore characterized by the lack of binding interaction between the protruding $\mathrm{OH}$ bond and the surface. In this respect the air water interface is also hydrophobic. By calibrating the signal at the free $\mathrm{OH}$ resonance we concluded that $25 \%$ of surface water molecules at this interface have one free $\mathrm{OH}$ bond [3].
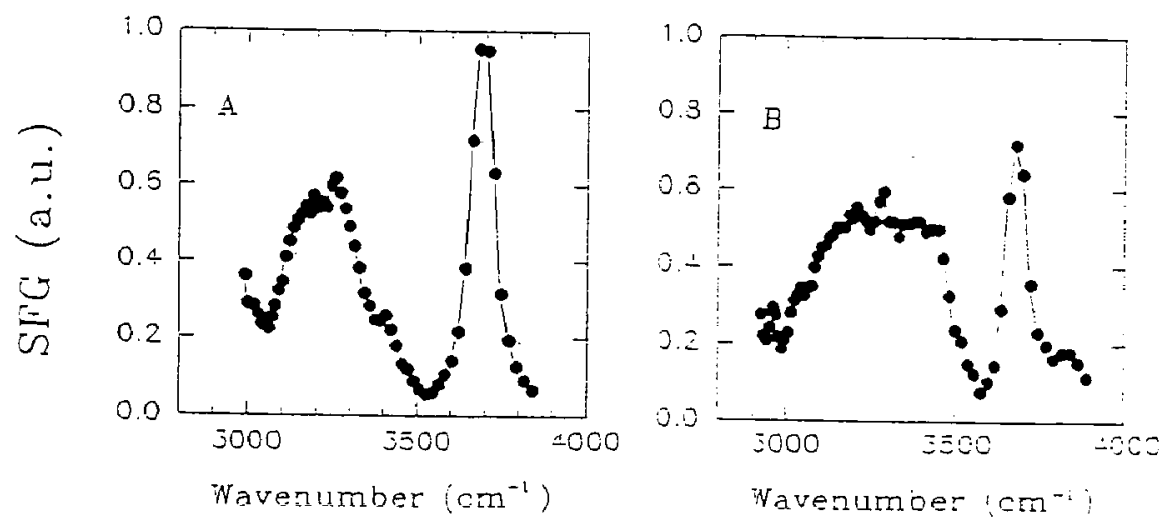

Figure 4: Spectrum of hydrophobic quartz-water (A) and hexane-water (B) interfaces 
However, we notice in fig 2 and 4 an appreciable change in the spectral feature due to hydrogen-bonded $\mathrm{OH}$ stretches between 3000 and $3600 \mathrm{~cm}^{-1}$. The peak at 3200 $\mathrm{cm}^{-1}$ is generally attributed to in-phase vibration of the coupled, symmetric $\mathrm{OH}$ stretch mode of the tetrahedrally coordinated water molecules. This peak dominates in the quartzice spectrum shown in Fig. 3 and is an indication of an ice like structure in the water molecular arrangement. The peak around $3400 \mathrm{~cm}^{-1}$ is still somewhat controversial but in any case represents bond disordering in the water molecular arrangement. As seen in Fig $4 \mathrm{a}$, the bonded $\mathrm{OH}$ spectrum part of the water-hydrophobic solid interface resembles the spectrum of the quartz-ice interface and indicates that water molecules at this hydrophobic interface are well bonded ordered. This result is in fair agreement with the prediction of recent molecular dynamic simulation [17]. The result can be understood from the physical consideration that the total number of hydrogen bonds should be maximized. This condition is satisfied when the water molecules form an ice like structure, with the surface terminated by dangling $\mathrm{OH}$ bonds. At the water-hydrophobic solid interface, the solid wall forces the surface water molecules into the more orderly ice-like structure because of the physical restriction involved in packing molecules against the wall.

The air-water interface, with about the same number of dangling bonds is about to follow the same model the difference is in the rigidity of the interface. The non rigid wall prevent the surface water molecules from being highly bond-ordered. This is reflected in the appearance of the pronounced bond-disordered peak at $3400 \mathrm{~cm}^{-1}$ in the spectrum of figure 2. The same is expected and is indeed observed for the liquid-liquid water-hexane interface presented on figure $4 \mathrm{~B}$.
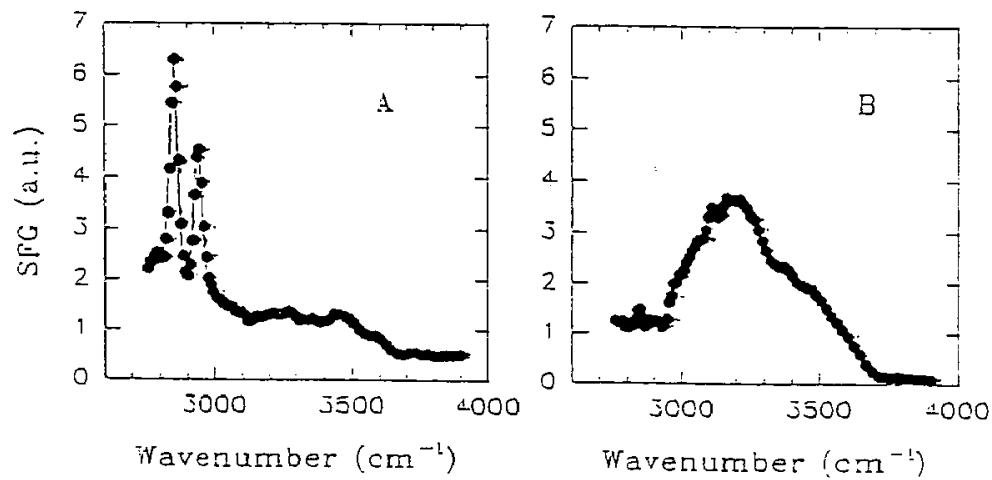

Figure 5: Spectrum of DMOAP coated quartz-air (A) and quartz-water (B) interfaces

We have also studied the interface between water and partly wettable solid substrate. The latter was prepared by absorbing on a fused quartz substrate a monolayer of DMOAP ( $\mathrm{n}$-octadecyldimethyl[3-(trimethoxysilyl)-propyl]ammoniumchloride), $\left.\mathrm{CH}_{3}\left(\mathrm{CH}_{2}\right)_{17}(\mathrm{Me})_{2} \mathrm{~N}^{+}\left(\mathrm{CH}_{2}\right)_{3} \mathrm{Si}(\mathrm{OMe}) \mathrm{Cl}^{-}\right)$. The head group of DMOAP is much larger than the cross section of the hydrocarbon chain, so that our quartz surface was covered with a layer of loosely packed hydrocarbon chains with a high density kink defects [18]. The SFG spectrum of the air-DMOAP-quartz interface is presented on Fig $6 \mathrm{~A}$. We notice the presence of two sharp peak around 2850 and $2940 \mathrm{~cm}-1$ which are respectively attributed to $\mathrm{CH}_{2}$ symmetric stretch mode and asymmetric $\mathrm{CH}_{2}$ stretch mode with perhaps some contribution from the Fermi resonance peak of the $\mathrm{CH}_{3}$ stretch mode. The broad but relatively weak spectral feature from 3000 to $3600 \mathrm{~cm}-1$ are presumably from water molecules absorbed on the surface. We notice an appreciable change in the waterDMOAP-quartz SFG spectrum shown on Fig.6b. The disappearance of the $\mathrm{CH}_{2}$ peak 
indicates that alkyl chain must have been straightened by interacting with water molecules. The enhancement of the spectral intensity of the hydrogen-bonded $\mathrm{OH}$ stretching modes results from the field oriented surface water molecules. This effect is establishment of a strong surface field due to ionized head group of DMOAP absorbed on the surface which orient several layer of water molecules at the interface and yield enhanced spectrum. This phenomena is similar to the behavior observed at the quartz water interface [2]. We have to notice the absence of discernible free $\mathrm{OH}$ peak. Than the partly wettable surface correspond to a situation in which the bulk water contact a surface partly covered by water (hydrophilic) and partly by hydrocarbon (hydrophobic).

We can now describe the hydrophobic interface at the molecular level. The surface water molecules does not interact strongly with the opposite surface. They form hydrogen bonding network, with dangling $\mathrm{OH}$ bonds. The large number of dangling $\mathrm{OH}$ bonds gives rise to a high interfacial surface energy. The hydrogen bonded network can be more or less ordered, depending on the oppositing hydrophobic surface. For partly wettable substrate prepared with DMOAP water molecules penetrates into the surfactant layer and straighten the alkyl chain.

\section{4- CONCLUSIONS}

We hope the examples described in this paper provide convincing evidence to the power of the SFG techniques as surface tools. In few years, SFG has been proven to be a versatile analytical tool for surface studies [18]. SFG is applicable for in-situ measurement to essentially all interfaces that are accessible to light. The technic has been demonstrated to be very sensitive, non destructive, and capable of remote sensing [18]. It ability for time resolved spectroscopy have also been demonstrated. In fact the technique is able to probe surface change with femtosecond time resolution [19]. This is certainly one of the most interesting issue because it allows us to investigate many interesting problems of surface dynamics and reaction in real time. The conjunction of this technique with surface force apparatus, near field and tunneling microscopy will allow to study with impressive time and spatial resolution fundamental processes at the molecular level and will provide a unique opportunity to look at surface science in a new light.

\section{References:}

[1] Du Q., Superfine R., Freysz E., Shen Y.R., Phys. Rev. Lett. 70 (1993) pp. 2313

[2] Du Q., Freysz E., Shen Y.R., Phys. Rev. Lett.7 2 (1994) pp. 238

[3] Du Q., Freysz E., Shen Y.R., Science 64 (1994) pp. 826

[4] Guyot Sionnest P., Hunt J.H., Shen Y.R., Phys. Rev. Lett. 59

(1987) pp. 1597

[5] Amstrong J.A., Blombergen N., Ducuing J., Pershan P.S., Phys. Rev. 127 (1962) pp. 1918

[6] Frenkel J., Kinetic theory of liquids (Dover, New York, 1955)

[7] Townsend R. M., Rice S. A., J. Chem. Phys. 94 (1991) pp. 2207;

Wilson M.A., Pohorille A., Pratt L.R., J. Chem. Phys. 91 (1987) pp.

4873 and reference therein

[8] Phillips M.C., "Water, A Comprehenive Treatise: The physics and physical chemistry of water", Vol. 5, Chap. 3

[9] Goh M.C. et al., J.Phys. Chem. 92 (1988) pp.5074 ; Goh M.C.,

Eisenthal K. B., Chem. Phys. Lett. 157 (1989) pp. 101

[10] Clark R.J.H., Hester R.E., "Advances in infrared and Raman

spectroscopy" ( Heyden, London, 1978), Vol. 5, Chap. 3

[11] Gavish M. et al. Science 250 (1990) pp.973

[12] Matsumoto M., Kataoka Y., J. Chem. Phys. 88 (1988) pp. 3233 
[13] Wisman W., Adv. Chem. Ser. 43 (1964) pp. 1; Whitesides G.M., Laibinis P.Z., Langmuir 6 (1960) pp. 87

[14] Israellachvili J.N., "Intermolecular and surface forces" ( Academic Press, London, 1989) Chaps 13-15

[15] Tanford C., "The hydrophobic effect" ( Wiley, New-York, ed. 2, 1980) pp. 1-35

[16] J. Sagiv, J. Am. Chem. Soc. 102 (1980) pp. 92

[17] Lee C.Y., McCammion J.A., Rossby R.J., J. Chem. Phys. 80 (1984) pp. 4448

[18] Y.R. Shen, Int. Conf. On Organic Materials for Nonlinear Optics, Oxford (England), June 29-30, (1988)

[19] Prybyla J.A., Tom H. W. K., Aumillier G.D., Phys. Rev. Lett. 68 (1992) pp. 503 